\title{
Analyzing effects of extra-virgin olive oil polyphenols on breast cancer-associated fatty acid synthase protein expression using reverse-phase protein microarrays
}

\author{
JAVIER A. MENENDEZ ${ }^{1,2,3}$, ALEJANDRO VAZQUEZ-MARTIN ${ }^{1,2,3}$, \\ CRISTINA OLIVERAS-FERRAROS ${ }^{1,2,3}$, ROCIO GARCIA-VILLALBA ${ }^{4}$, ALEGRIA CARRASCO-PANCORBO ${ }^{4}$, \\ ALBERTO FERNANDEZ-GUTIERREZ ${ }^{4}$ and ANTONIO SEGURA-CARRETERO ${ }^{4}$
}

${ }^{1}$ Catalan Institute of Oncology, (ICO-Girona)-Health Services Division of Catalonia; ${ }^{2}$ Girona Biomedical Research Institute (IdIBGi); ${ }^{3}$ Medical Oncology, Dr. Josep Trueta University Hospital of Girona, Girona, Catalonia; ${ }^{4}$ Department of Analytical Chemistry, Faculty of Sciences, University of Granada, Granada, Spain

Received June 5, 2008; Accepted July 28, 2008

DOI: 10.3892/ijmm_00000039

\begin{abstract}
Inhibitors of fatty acid synthase (FASN), a key enzyme involved in the anabolic conversion of dietary carbohydrates to fat in mammals, are receiving increasingly more attention as they may provide therapeutic moieties for the treatment of human malignancies. Natural compounds, such as the green tea polyphenol epigallocatechin-3-gallate, have been shown to induce anti-cancer effects by suppressing FASN, which may account for the epidemiologically observed inverse correlation between green-tea drinking and cancer risk in Oriental populations. Since extra-virgin olive oil (EVOO)-derived phenolics have been suggested to possess biological activities that may explain the health-promoting effects of the 'Mediterranean diet', we evaluated their effects on the expression of FASN protein in human breast epithelial cell lines. First, we developed a reverse phase protein microspot array (RPPA) capable of rapidly assessing the relative amount of FASN protein in whole lysates from cultured human cells. Then we tested the effects of phenolic fractions from EVOO and its main constituents including single phenols (i.e. tyrosol, hydroxytyrosol, vanillin), phenolic acids (i.e. caffeic acid, $p$-coumaric acid, vanillic acid, ferulic acid, elenolic acid), lignans (i.e. 1-[+]-pinoresinol,
\end{abstract}

Correspondence to: Dr Javier A. Menendez, Catalan Institute of Oncology-Girona (ICO-Girona), Girona Biomedical Research Institute (IdIBGi), Medical Oncology, Dr. Josep Trueta University Hospital of Girona, Avenida de Francia s/n, E-17007 Girona, Catalonia, Spain

E-mail: jmenendez@ico.scs.es; jmenendez@iconcologia.net

Dr Antonio Segura-Carretero, Research Group FQM-297, Department of Analytical Chemistry, Faculty of Sciences, University of Granada, C/Fuentenueva s/n, E-18071 Granada, Spain E-mail: ansegura@ugr.es

Key words: olive oil, polyphenols, cancer, fatty acid synthase, human epidermal growth factor receptor 2 1-[+]-acetoxy-pinoresinol), flavonoids (i.e. apigenin, luteolin), or secoiridoids (i.e. deacetoxyoleuropein aglycone, ligstroside aglycone, oleuropein glycoside, oleuropein aglycone) on FASN protein expression. EVOO polyphenols lignans, flavonoids and secoiridoids were found to drastically suppress FASN protein expression in HER2 gene-amplified SKBR3 breast cancer cells. Equivalent results were observed in MCF-7 cells engineered to overexpress the HER2 tyrosine kinase receptor, a well-characterized up-regulator of FASN expression in aggressive sub-types of cancer cells. EVOOderived lignans, flavonoids and secoiridoids were significantly more effective than the mono-HER2 inhibitor trastuzumab ( $\sim 50 \%$ reduction) and as effective as the dual HER1/HER2 tyrosine kinase inhibitor lapatinib ( $\geq 95 \%$ reduction) at suppressing high-levels of FASN protein in HER2overexpressing SKBR3 and MCF-7/HER2 cells. EVOO single phenols and phenolic acids failed to modulate FASN expression in SKBR3 and MCF-7/HER2 cells. These findings reveal for the first time that phenolic fractions, directly extracted from EVOO, may induce anti-cancer effects by suppressing the expression of the lipogenic enzyme FASN in HER2-overexpressing breast carcinoma cells, thus offering a previously unrecognized mechanism for EVOO-related cancer preventive effects.

\section{Introduction}

The implications of Fatty Acid Synthase (FASN)-catalyzed terminal steps in the de novo biogenesis of long-chain fatty acids (FAs) did not become a focus of interest in the cancer field until the early 1990s. In 1994, Kuhajda and colleagues unambiguously identified, through sequence homology and enzymology, the oncogenic antigen-519 (OA-519), a molecule found in tumor cells from breast cancer patients with poor prognosis, as FASN. Since then, overexpression and increased activity of FASN have been recognized as some of the most frequent molecular alterations in cancer cells (reviewed in refs. 1-4). Thus, immunohistochemical studies have reported extremely high levels of FASN in many human 
epithelial cancers and their pre-neoplastic lesions, including breast, colorectum, prostate, bladder, ovary, oesophagus, stomach, lung, oral tongue, oral cavity, head and neck, thyroid and endometrium, and also in mesothelioma, nephroblastoma, retinoblastoma and soft tissue sarcomas. More recently, FASN overexpression has also been described in patients with invasive or micro-invasive Paget's disease of the vulva, in cutaneous melanocytic neoplasms including melanoma, and in patients with hepatocellular or pancreatic carcinomas. Importantly, high-levels of FASN-catalyzed de novo FA biosynthesis positively correlate with aggressive behaviors, poorer prognoses, and increased risk of death in various cancers. As FASN-related 'lipogenic phenotype' emerges early in carcinogenesis, it might represent a possible target in cancer prevention and/or in retarding the invasive progression in early lesions. At later stages, FASN represents a valuable drug target for treatment of advanced/metastatic carcinomas. The classic consideration of the lipogenic phenotype (i.e. activation of the genetic program involved in de novo FA synthesis including lipogenic enzymes and key metabolic regulators) as a minor anabolic-energy-storage pathway is not valid any longer in the breast cancer setting. Rather, endogenous FA biogenesis catalyzed by lipogenic enzymes such as FASN may represent a previously unrecognzied oncogenic stimulus that drives progression of human breast epithelial cell toward malignancy (4).

Since the pioneering observation that inhibition of FASN activity by the mycotoxin cerulenin preferentially kills cancer cells and retards the growth of tumors in xenograft models, numerous in vitro and in vivo studies have confirmed the potential of FASN as a target for antineoplastic intervention (reviewed in ref. 5). Other FASN inhibitors such as the cerulenin derivative $\mathrm{C} 75$, the $\mathrm{B}$-lactone orlistat, and the antibiotic triclosan, have been identified and have been demonstrated to limit cancer cell growth by inducing apoptotic cell death. Although the potential of FASN as a target for antineoplastic intervention has eventually been confirmed by RNA interference (RNAi)-knockdown of FASN, few of the currently available FASN inhibitors are 'exclusively' selective for their FASN target. Natural compounds such as the green tea polyphenol epigallocatechin-3-gallate (EGCG) and other naturally occurring flavonoids have been shown to induce anti-cancer effects by suppressing FASN activity and/or expression, which may account for the epidemiologically observed inverse correlation between green-tea drinking and cancer risk in Oriental populations (6-12). Similarly, extravirgin olive oil (EVOO)-derived phenolics have been suggested to possess biological activities that may explain the beneficial health properties of the 'Mediterranean diet' (13-19). Three main classes of phenolic compounds are represented in different proportions in EVOO: simple phenols (phenolic acids and hydrolyzed secoiridoids such as hydroxytyrosol, tyrosol and elenolic acid), secoiridoids and lignans (13-17). Since phenols and polyphenols have well-established roles in disease prevention whereas the biological effects of these dietary phytochemicals are varied and compound-specific (20), the aim of the present study was to determine the specific ability of EVOO phenolics to suppress the biosynthesis of FASN oncoprotein (Fig. 1). Using HER2 gene-amplified SKBR3 breast cancer cells; a naturally occurring paradigm in which up to $28 \%$ of cytosolic protein weight is constituted by FASN- and MCF-7 cells engineered to overexpress the HER2 tyrosine kinase receptor, EVOO-derived phenols were investigated on their ability to modulate FASN protein expression. Our current findings reveal for the first time that EVOO secoiridoids and lignans strongly inhibit the expression of FASN oncoprotein in HER2-overexpressing breast cancer cells, thus providing a plausible molecular candidate that might explain, at least in part, the ability of EVOO-rich diets to promote anti-cancer effects in a biological subset of HER2positive breast carcinomas (21).

\section{Materials and methods}

Materials. A 50/50 mixture of two commercial EVOO samples (i.e. Picual and Arbequina Spanish varieties) was used for this study. Solid-phase extraction (SPE) followed by semi-preparative high-performance liquid chromatography (HPLC) was used to isolate polyphenolic fractions from commercial EVOO as previously described (22-27). Analytical capillary electrophoresis coupled to mass spectrometry (CEMS) was performed to ascertain the composition of the isolated fractions and to confirm their molecular identity. Trastuzumab (Herceptin ${ }^{\mathrm{TM}}$ ), kindly provided by Hospital Universitari de Girona Dr. Josep Trueta Pharmacy (Girona, Spain), was solubilized in bacteriostatic water for injection containing $1.1 \%$ benzyl alcohol (stock solution at $21 \mathrm{mg} / \mathrm{ml}$ ), stored at $4{ }^{\circ} \mathrm{C}$ and used within one month. Lapatinib (GW572016; Tykerb ${ }^{\circledR}$ ) was kindly provided by GlaxoSmithKline (GSK), Corporate Environment, Health \& Safety (Brentford, Middlesex, UK). Lapatinib was dissolved in DMSO and stored in the dark as stock solution $(10 \mathrm{mM})$ at $-20^{\circ} \mathrm{C}$ until utilization. For experimental use, EVOO phenolics, trastuzumab and lapatinib and were prepared freshly from stock solutions and diluted with growth medium. Control cells were cultured in a medium containing the same concentration $(\mathrm{v} / \mathrm{v})$ as the experimental cultures with treatments. The vehicle solutions had no noticeable influence on the proliferation of experimental cells.

Cell lines and culture conditions. SKBR3 and MCF-7 breast cancer cell lines were obtained from the American Type Culture Collection (ATCC) and they where routinely grown in Dulbecco's modified Eagle's medium (DMEM, Gibco) containing $10 \%$ heat-inactivated foetal bovine serum (FBS, Bio-Whittaker), $1 \%$ L-glutamine, $1 \%$ sodium pyruvate, $50 \mathrm{U} / \mathrm{ml}$ penicillin and $50 \mu \mathrm{g} / \mathrm{ml}$ streptomycin. Cells were maintained at $37^{\circ} \mathrm{C}$ in a humidified atmosphere of $95 \%$ air and $5 \% \mathrm{CO}_{2}$. Cells were screened periodically for Mycoplasma contamination.

Construction of $p B A B E / H E R 2$ retroviruses and retroviral infection of MCF-7 cells. A full-length human HER2 cDNA construct in the pCMV-SPORT6 plasmid was purchased from RZPD (Berlin, Germany). The insert was excised from pCMV-SPORT6 using EcoRV and NotI sites and blunt end ligated into the pBABE-puro retroviral vector (Addgene) at the EcoRI site. Retroviruses were generated by the cotransfection of 293T-derived phoenix cells with the retroviral constructs (pBABE, pBABE-HER2) and the packaging vector pCL-Eco by using FuGene transfection reagent 


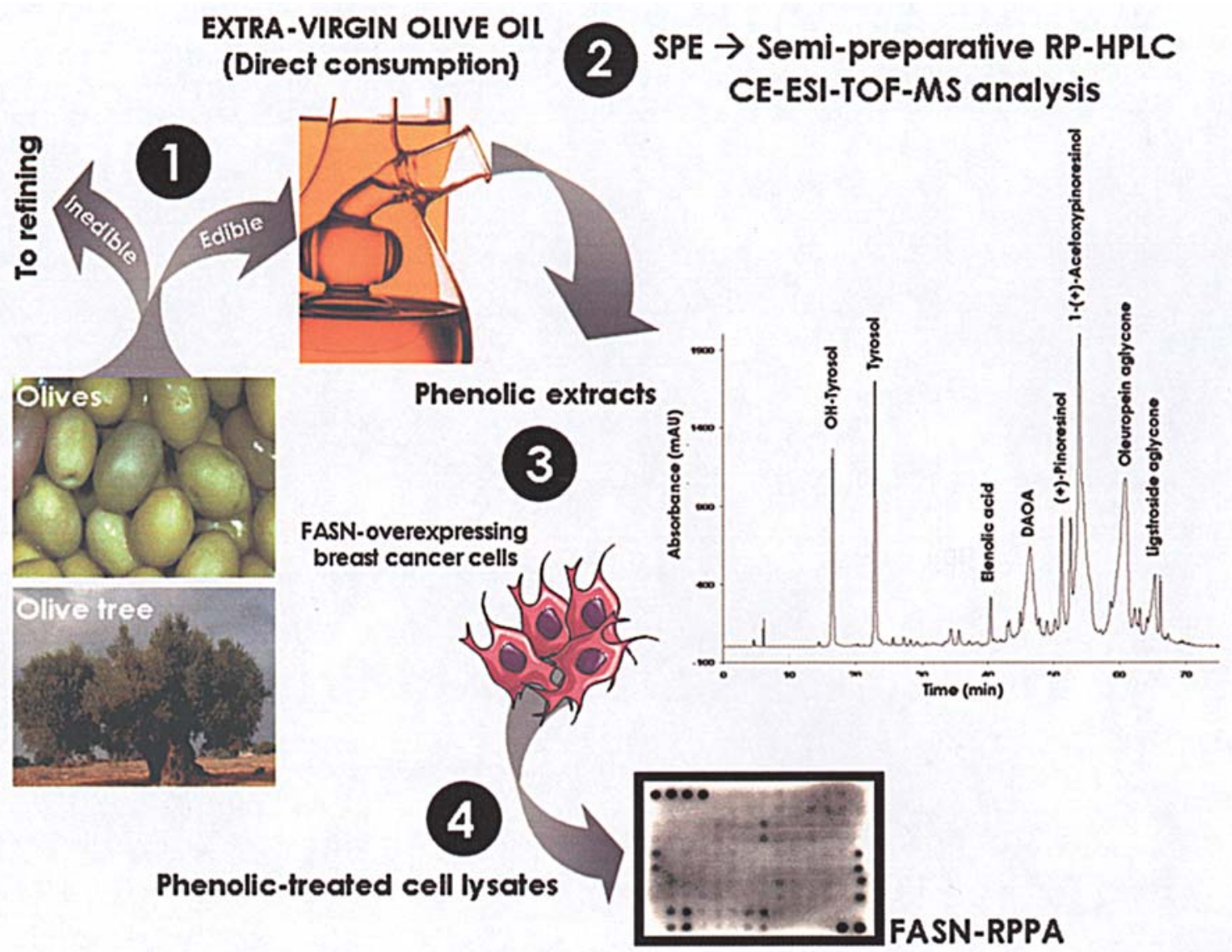

Figure 1. Sample, extraction, isolation and analysis of the anti-FASN effects of EVOO-derived phenolic fractions. EVOO is unique among vegetable oils due to its high level of phenolic compounds. These levels are possible as EVOO is obtained from the olive fruit (Olea Europea L.) solely by mechanical means, without further treatment other than washing, filtration, decantation or centrifugation (1). Since synthesis of EVOO phenolics is currently not practical, we used solid phase extraction (SPE) with Diol-cartridges followed by semi-preparative reverse phase (RP)-HPLC to isolate different fractions of phenols from the EVOO. We employed capillary electrophoresis (CE)-electrospray ionization (EI)-mass spectrometry (CE-ESI-MS) methodology to establish the composition of the isolated fractions. Identification of the compounds was obtained from the accurate mass and the isotopic pattern of the peaks by applying ESI-time of flight (TOF)-MS analyzer (2). For experimental use, EVOO phenolics were dissolved in 100\% ethanol (stock solutions). For culture treatment, EVOO phenolics were prepared freshly from stock solutions and diluted with growth medium (3). Detection of FASN expression in array format using whole cell lysates following treatments with EVOO phenolics was performed as described in Fig. 2 (see Materials and methods) (4).

(Roche Diagnostics, Barcelona, Spain) and $5 \mu \mathrm{g}$ of each plasmid per $0.5 \times 10^{6}$ cells. 293T Cells were cultured at $5 \%$ $\mathrm{CO}_{2}, 37^{\circ} \mathrm{C}$ in DMEM containing $10 \%(\mathrm{v} / \mathrm{v})$ heat-inactivated FBS. After 48 h, medium conditioned by transfected $293 \mathrm{~T}$ cells was filtered and immediately added to MCF-7 cells in the presence of $4 \mu \mathrm{g} / \mathrm{ml}$ polybrene (Sigma-Chemicals, St. Louis, MO, USA). At 48 h following infection, MCF-7/ pBABE and MCF-7/HER2 cells were selected by using 2.5 $\mu \mathrm{g} / \mathrm{ml}$ puromycin for $72 \mathrm{~h}$. Expression of virally encoded HER 2 protein was confirmed by HER2-specific ELISA analyses.

Treatments. To assess the effects of EVOO phenolics and anti-HER2 agents on FASN protein expression, cells were plated in 100-mm tissue culture dishes and cultured in DMEM with $10 \%$ FBS until they achieved $75-80 \%$ confluence. The cells were washed twice with serum-free DMEM, and incubated overnight in serum-free DMEM. Cells were then cultured for $48 \mathrm{~h}$ in low-serum $(0.1 \%$ FBS $)$ DMEM in the presence or absence of experimental agents, as specified. After treatment, cells were washed twice with cold-PBS and then lysed in buffer (20 mM Tris pH 7.5, $150 \mathrm{mM} \mathrm{NaCl}, 1 \mathrm{mM}$ EDTA,
$1 \mathrm{mM}$ EGTA, $1 \%$ Triton X-100, $2.5 \mathrm{mM}$ sodium pyrophosphate, $1 \mathrm{mM}$ ß-glycerolphosphate, $1 \mathrm{mM} \mathrm{Na}_{3} \mathrm{VO}_{4}, 1 \mu \mathrm{g} / \mathrm{ml}$ leupeptin, $1 \mathrm{mM}$ phenylmethylsulfonyl-fluoride) for $30 \mathrm{~min}$ on ice. The lysates were cleared by centrifugation in an Eppendorff tube $\left(15 \mathrm{~min}\right.$ at $\left.14,000 \mathrm{xg}, 4^{\circ} \mathrm{C}\right)$. Protein content was determined against a standardized control using the Pierce Protein Assay Kit.

Preparation of reverse-phase protein microspot arrays. A template of 120 spots (12 spots by 10 spots) covering an area of $48 \mathrm{~cm}^{2}(6 \times 8 \mathrm{~cm})$ was generated from a computer. Hybond ECL membranes (Amersham Pharmacia Biotech.) were placed on the top of the template. Through the white light box, the dark spots in the template were clearly visualized from the membranes and used as a guide to spot solution onto the membranes. Quantities of $0.25 \mu 1$ of cell lysates $(2 \mu \mathrm{g}$ total protein/sample) were manually loaded onto membranes using a 2- $\mu 1$ Pipetman in duplicate. After blocking with 5\% bovine serum albumin (BSA)/TBS (0.01 M Tris-HCl, pH $7.6 / 0.15 \mathrm{M} \mathrm{NaCl}$ ) for $1 \mathrm{~h}$ at room temperature (RT), membranes were incubated with a mouse anti-FASN monoclonal antibody (1:1000 dilution of clone 23, BD Biosciences Pharmingen, 
a

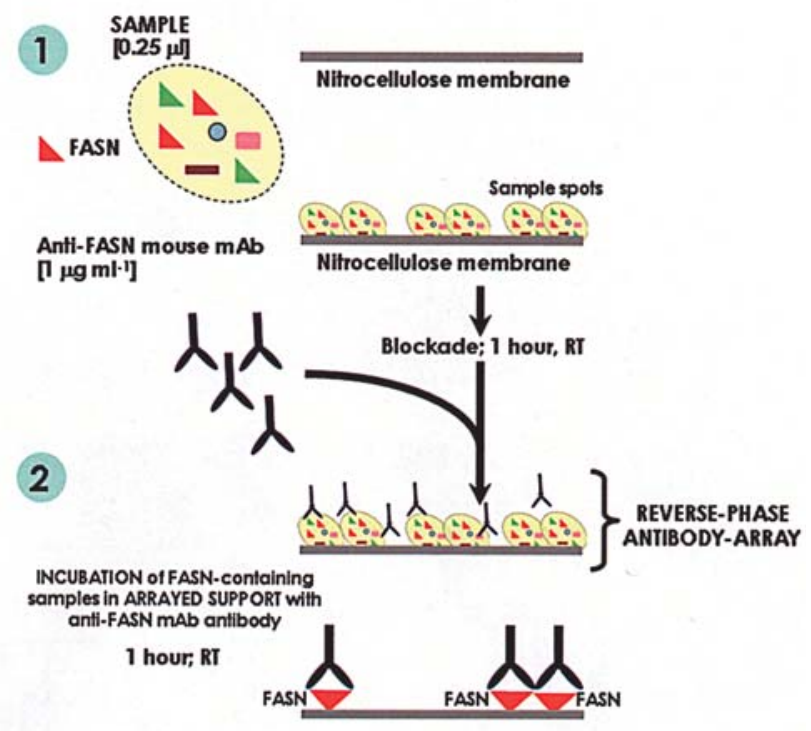

3
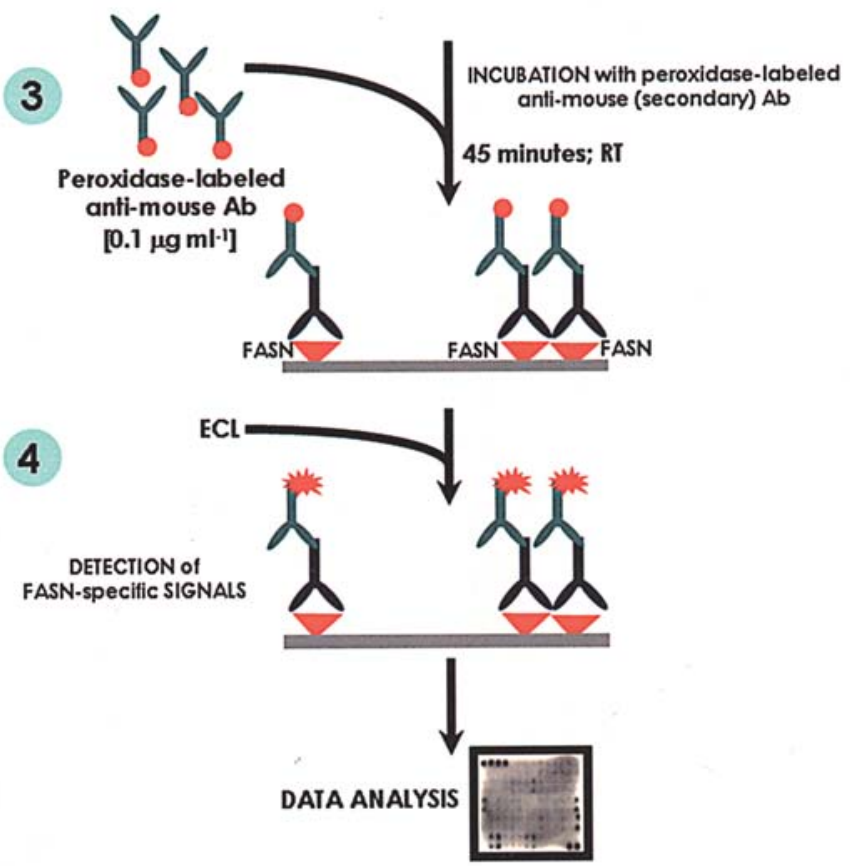

Figure 2. a) Design of FASN-specific reverse-phase protein arraying (FASN-RPPA). Experimental cell populations are lysed and manually arrayed onto nitrocellulose membranes with a $2-\mu 1$ Pipetman in duplicate (1). After arraying, the membranes are incubated with an anti-FASN monoclonal antibody (2) that is detected by a chemiluminescent assay (3). Intensity of the signal is proportional to the concentration of the target protein (4). b) Detection of FASN expression in array format. Several dilutions of SKBR3-derived whole cell lysates were arrayed, processed and developed as described (see Materials and methods). Figures show a representative FASN-RPPA $(n=3)$.

San Diego, CA, USA), which functioned as 'detector antibody'. Following incubation for $1 \mathrm{~h}$, the excess antibody was washed out and goat anti-mouse immunoglobulin G conjugated to HRP (1:10,000 dilution), which binds to 'detector antibody', was added for $45 \mathrm{~min}$. Signals were developed by an ECL system and their intensities were scanned by Scion densitometry. b

[Cell lysate] $\mu \mathrm{g} \mu$ ]

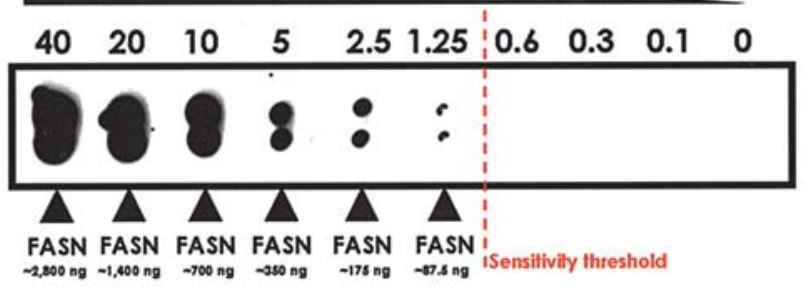

Results

Development of a FASN-specific reverse-phase protein microspot array (FASN-RPPA). We first developed a reversephase protein microspot array (RPPA) capable of rapidly assessing the relative amount of FASN protein in whole lysates from cultured human breast cancer cells (Fig. 2). To validate FASN-specific RPPAs, $0.25 \mu 1$ of serial dilutions from wholecell extracts of the FASN-overexpressing SKBR3 cell line were spotted in an array configuration (in duplicate) onto ECL nitrocellulose membranes. These applications result in 0.2-0.3 mm-wide spots containing the whole cellular protein repertoire corresponding to a given pathological state. The term 'reverse-phase' is derived from the fact that this type of protein microarray immobilises the protein to be analysed. This is in contrast to conventional protein arrays that immobilise the antibody probe (28-30). Subsequently, the membrane was incubated with a mouse anti-FASN monoclonal antibody that functioned as a 'detector' antibody as RPPA denatures the protein lysates to immobilisation and thus does not require labeling of the protein to be analysed. Since individually targeted protein entity (i.e. FASN) is expected to be present in very low concentrations, an amplification system had to be developed that allows for the specific and sensitive detection of the protein of interest. Here, 'FASN spots' were detected by amplified chemiluminescent assays following incubation with a goat anti-mouse immunoglobulin G conjugate to HRP (Fig. 2).

EVOO polyphenols, secoiridoids, lignans and flavonoids, but not EVOO single phenols and phenolic acids, inhibit FASN protein expression in breast epithelial cells. To elucidate the effects of EVOO phenolics on the expression levels of FASN protein, SKBR3 cells were cultured in low-serum (0.1\% FBS) medium in the absence or presence of an equimolar concentration of EVOO phenolics (i.e., $50 \mu \mathrm{M}$ ). After incubation for $48 \mathrm{~h}$, the cells were harvested and equivalent quantities of cell lysates ( $2 \mu \mathrm{g}$ of total protein in a volume of $0.25 \mu \mathrm{l} / \mathrm{sample}$ ) were spotted onto the microspot array in duplicate as previously described. Single phenols (i.e. tyrosol, hydroxytyrosol and vanillin) and phenolic acids (i.e. caffeic acid, $p$ coumaric acid, vanillic acid, ferulic acid and elenolic acid) failed to significantly decrease FASN protein expression compared with untreated control cells. A moderate to drastic reduction in FASN protein levels was observed in whole lysates of SKBR3 cells cultured in the presence of the EVOO secoiridoids deacetoxyoleuropein aglycone (45-50\% reduction), ligstroside aglycone ( $\sim 80-85 \%$ reduction), oleuropein 

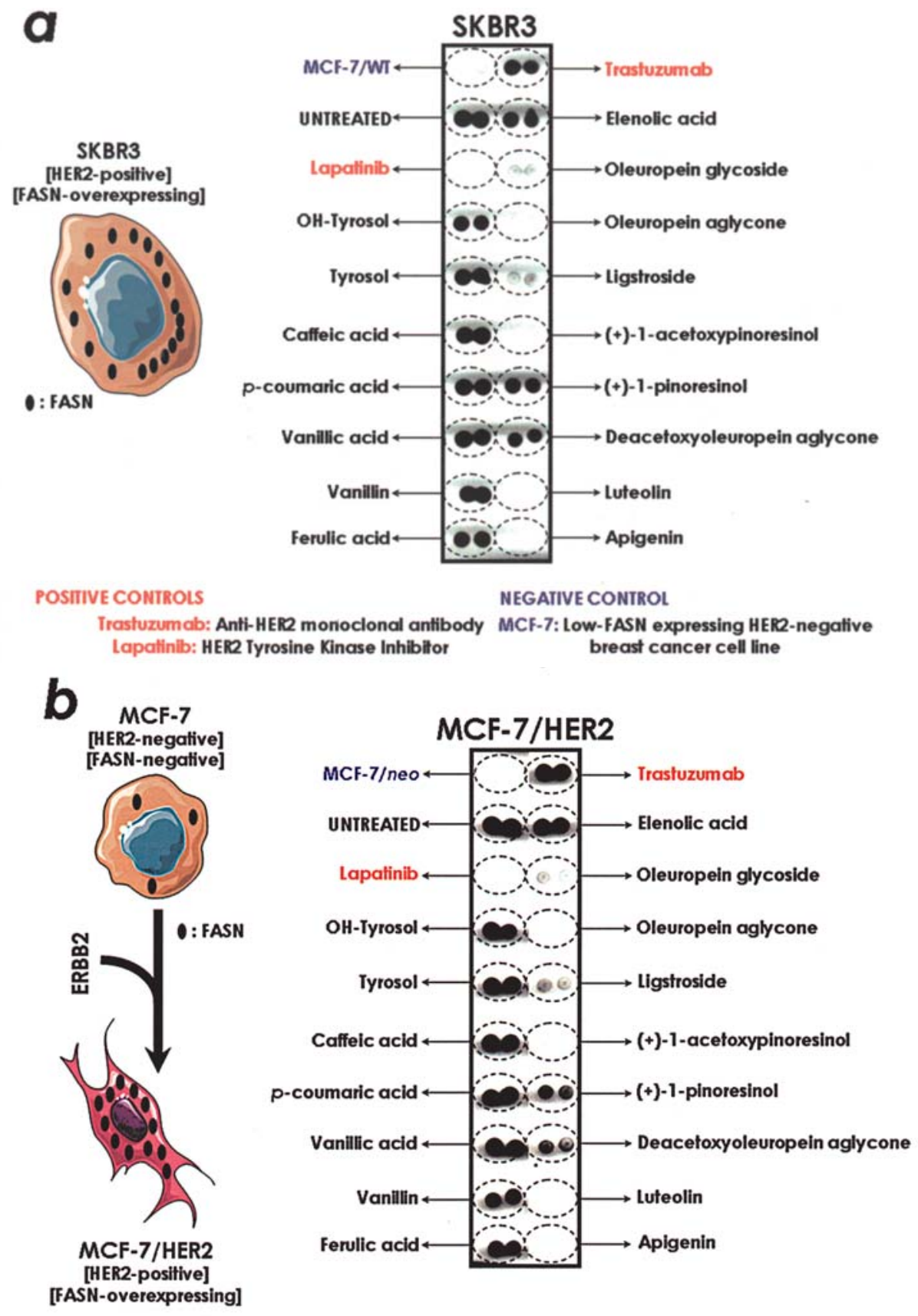

Figure 3. Effects of EVOO phenolics on FASN protein expression. Protein ( $2 \mu \mathrm{g})$ from SKBR3 a) and MCF-7/HER2 b) whole cell lysates obtained following culture in the absence of EVOO phenolics, trastuzumab and lapatinib were arrayed, processed and developed as described in Materials and methods. A representative FASN-RPPA $(n=3)$ is shown.

glycoside ( $\sim 85-90 \%$ reduction), and oleuropein aglycone (100\% reduction). The EVOO lignan 1-[+]-acetoxypinoresinol was significantly more effective at reducing FASN protein levels than 1-[+]-pinoresinol ( $\sim 100 \%$ reduction versus $30-35 \%$ reduction, respectively). EVOO flavonoids, apigenin and luteolin, completely suppressed ( $100 \%$ reduction) FASN protein expression in SKBR3 cells (Fig. 3a).

Overexpression of the HER2 oncogene is a well recognised oncogenic mechanism that leads to constitutive up-regulation of the lipogenic enzyme FASN $(31,32)$. However, FASN overexpression is sufficient to trigger a breast cancer-like phenotype functionally dependent in HER2 activity $(33,34)$. Therefore, we finally evaluated the ability of EVOO phenolics to regulate FASN expression in breast cancer cells engineered to overexpress HER2 (Fig. 3b). FASN-RPPAs likewise detected enormous amounts of FASN protein in whole cell lysates from MCF-7/HER2 cells when compared with HER2negative MCF-7 parental cells. Similarly to the regulatory effects observed in SKBR3 cells, EVOO single phenols and phenolic acids (i.e. tyrosol, hydroxytyrosol, vanillin, caffeic acid, $p$-coumaric acid, vanillic acid, ferulic acid, and elenolic acid) failed to down-regulate FASN protein expression in MCF-7/HER2 cells. A significant decrease in FASN protein levels was observed in the whole lysates of MCF-7/HER2 cells cultured in the presence of the EVOO secoiridoids deacetoxyoleuropein aglycone (50-55\% reduction), ligstroside 
aglycone ( $\sim 85-90 \%$ reduction), oleuropein glycoside ( $\sim 90-95 \%$ reduction), and oleuropein aglycone ( $\sim 100 \%$ reduction). The EVOO lignan 1-[+]-pinoresinol decreased FASN levels more efficiently in MCF-7/HER2 ( $~ 50 \%$ reduction) than in SKBR3 (30-35\% reduction, respectively). The EVOO lignan $1-[+]$-acetoxypinoresinol and the EVOO flavonoids, apigenin and luteolin, completely suppressed (100\% reduction) FASN protein expression in MCF-7/HER2 cells (Fig. 3a).

Parallel experiments were performed in the presence of the mono-HER 2 monoclonal antibody trastuzumab (Herceptin $^{\text {TM }}$ ) and of the dual-HER1/HER2 tyrosine kinase inhibitor lapatinib $\left(\right.$ Tykerb $\left.^{\circledR}\right)$. Remarkably, EVOO-derived lignans, flavonoids and secoiridoids were significantly more effective than trastuzumab ( $\sim 50 \%$ reduction) and as effective as lapatinib ( $\geq 95 \%$ reduction) at suppressing high-levels of FASN protein in HER2-overexpressing SKBR3 and MCF-7/ HER 2 cells.

\section{Discussion}

Although inhibitors of FASN are receiving increasingly more attention together with the experimental and clinical evidence suggesting that FASN is a candidate gene for the pathophysiology not only of cancer disease but of human obesity and obesity-related metabolic disorders, most previous studies on FASN inhibitors have been confined to their inhibitory effects on the enzymatic activity of FASN (5). However, few of these FASN blockers are expected to be 'exclusively' selective for FASN while the combination of FASN structural complexity, and, until recently the lack of X-ray crystallography data of mammalian FASN created a significant challenge in the exploitation of FASN as a valuable target for drug development (4). Therefore, it would be more useful to investigate the inhibitory effects of novel compounds on FASN protein expression as the molecular relevance of FASN as a target for anti-neoplastic and anti-obesity measures has been confirmed by RNAi-knockdown of FASN in cultured cancer and adipose cells, respectively. On the basis of these considerations, we focused on the effects that EVOO phenolics may exert on the FASN protein levels in human breast cancer cells.

Earlier studies demonstrated that the green tea polyphenol EGCG could down-regulate FASN expression by suppressing EGFR (epidermal growth factor receptor; HER1) signaling and downstream phosphatidylinositol 3-kinase (PI3K)/AKT activation in the MCF-7 breast cancer cell line. EGCG was found also to efficiently suppress heregulin- $\$ 1$-induced FASN expression by inhibiting PI3K/AKT and mitogen-activated protein kinase (MAPK) cascade signaling in human breast cancer cells (6-11). Here, we examined the effects of EVOO phenolics when FASN protein expression was modulated by another member of the HER tyrosine kinase receptors network (i.e. HER2). Remarkably, EVOO-derived lignans, flavonoids and secoiridoids were found to decrease FASN protein content in HER2-overexpressing breast cancer cells more effectively than the mono-HER2 inhibitor trastuzumab. EVOO polyphenols were as effective as the dual-HER1/HER2 tyrosine kinase inhibitor lapatinib at suppressing high-levels of FASN protein in SKBR3 and MCF-7/HER2 breast cancer cells.

FASN has been implicated in the natural history of breast cancer largely through its connection to the activity and/or expression of key cancer-related oncogenes such as the Type I receptor tyrosine kinase HER2 (2-4). Transcriptomic, proteomic, and functional analyses have demonstrated that HER2 overexpression leads to constitutive up-regulation and maintenance of an exacerbated endogenous FA biogenesis, a 'lipogenic benefit' in terms of enhanced breast cancer cell proliferation, survival, chemoresistance and metastasis. Disturbance of the lipogenic phenotype rapidly switches off the oncogenic activity of the HER2 signaling platform, ultimately resulting in apoptotic tumor cell death. The sole activation of endogenous FA biosynthesis in non-cancerous breast epithelial cells is sufficient to induce a breast cancer-like phenotype functionally dependent on HER 2 activity. Thus, it is reasonable to suggest that FASN-catalyzed endogenous lipogenesis represents a druggable 'Achilles heel' in HER2driven oncogenesis that should open a new window of opportunity for metabolically preventing and/or treating HER2-positive breast carcinomas. The suppression of FASN preferentially leads to growth inhibition and cell apoptosis in HER2-positive cultured breast cancer cells and xenografts $(2,5,31,35)$. Our current findings reveal for the first time, to the best of our knowledge, that EVOO polyphenols (i.e. lignans, secoiridoids, and flavonoids) drastically suppress the expression of FASN protein in HER2-overexpressing breast cancer cells. It remains to be elucidated whether EVOO phenolic-induced blockade of HER2 activity is sufficient to explain their ability to suppress FASN expression. Auspiciously, we recently presented evidence demonstrating that the aglycone form of oleuropein directly down-regulates HER2 expression and tyrosine kinase activity cultured HER2overexpressing breast cancer cells (18). Studies are currently underway in our laboratory to ultimately determine the antiHER2 tyrosine kinase effects of EVOO polyphenols such as the lignans (+)-pinoresinol and (+)-1-acetoxypinoresinol and the secoiridoids deacetoxy-oleuropein aglycone and ligstroside aglycone.

In summary, naturally occurring EVOO polyphenols (e.g. secoiridoids, lignans, and flavonoids), through their previously unrecognized ability to suppress the oncoprotein FASN, have the potential to be useful in the chemoprevention and/or treatment of breast carcinomas in which FASN overexpression results from HER2-driven oncogenic signaling. Moreover, these findings offer a novel molecular mechanism through which EVOO-rich dietary patterns can protect against HER2positive breast carcinomas (21).

\section{Acknowledgments}

Javier A. Menendez is the recipient of a Basic, Clinical and Translational Research Award (BCTR0600894) from the Susan G. Komen Breast Cancer Foundation (TX, USA). This work was supported in part by Instituto de Salud Carlos III (Ministerio de Sanidad y Consumo, Fondo de Investigación Sanitaria -FIS-, Spain, grants CP-05-00090, PI06-0778 and RD06-0020-0028 to Javier A. Menendez). Javier A. Menendez and Antonio Segura-Carretero are also supported by a grant from the Fundación Científica de la Asociación Española Contra el Cáncer (AECC, Spain) and by the Ministerio de Educación y Ciencia CTQ2005-01914/BQU and Junta de Andalucía (Proyecto de Excelencia AGR-02619). 


\section{References}

1. Kuhajda FP: Fatty-acid synthase and human cancer: new perspectives on its role in tumor biology. Nutrition 16: 202-208, 2000.

2. Menendez JA, Lupu R and Colomer R: Targeting fatty acid synthase: potential for therapeutic intervention in Her-2/neuoverexpressing breast cancer. Drug News Perspect 18: 375-385, 2005.

3. Menendez JA and Lupu R: Oncogenic properties of the endogenous fatty acid metabolism: molecular pathology of fatty acid synthase in cancer cells. Curr Opin Clin Nutr Metab Care 9: 346-357, 2006

4. Menendez JA and Lupu R: Fatty acid synthase and the lipogenic phenotype in cancer pathogenesis. Nat Rev Cancer 7: 763-777, 2007.

5. Lupu R and Menendez JA: Pharmacological inhibitors of Fatty Acid Synthase (FASN)-catalyzed endogenous fatty acid biogenesis: A new family of anti-cancer agents? Curr Pharm Biotechnol 7: 495-502, 2006.

6. Yeh CW, Chen WJ, Chiang CT, Lin-Shiau SY and Lin JK: Suppression of fatty acid synthase in MCF-7 breast cancer cells by tea and tea polyphenols: a possible mechanism for their hypolipidemic effects. Pharmacogenomics J 3: 267-276, 2003.

7. Wang X, Song KS, Guo QX and Tian WX: The galloyl moiety of green tea catechins is the critical structural feature to inhibit fatty-acid synthase. Biochem Pharmacol 66: 2039-2047, 2003.

8 Brusselmans K, Vrolix R, Verhoeven G and Swinnen JV: Induction of cancer cell apoptosis by flavonoids is associated with their ability to inhibit fatty acid synthase activity. J Biol Chem 280: 5636-5645, 2005.

9. Tian WX: Inhibition of fatty acid synthase by polyphenols. Curr Med Chem 13: 967-977, 2006

10. Lin JK and Lin-Shiau SY: Mechanisms of hypolipidemic and anti-obesity effects of tea and tea polyphenols. Mol Nutr Food Res 50: 211-217, 2006.

11. Pan MH, Lin CC, Lin JK and Chen WJ: Tea polyphenol (-)epigallocatechin 3-gallate suppresses heregulin-beta1-induced fatty acid synthase expression in human breast cancer cells by inhibiting phosphatidylinositol 3-kinase/Akt and mitogenactivated protein kinase cascade signaling. J Agric Food Chem 55: 5030-5037, 2007

12. Puig T, Vázquez-Martín A, Relat J, Pétriz J, Menéndez JA, Porta R, Casals G, Marrero PF, Haro D, Brunet J and Colomer R: Fatty acid metabolism in breast cancer cells: differential inhibitory effects of epigallocatechin gallate (EGCG) and C75. Breast Cancer Res Treat 109: 471-479, 2008.

13. Visioli F, Bellomo $\mathrm{G}$ and Galli $\mathrm{C}$ : Free radical-scavenging properties of olive oil polyphenols. Biochem Biophys Res Commun 247: 60-64, 1998.

14. Visioli F and Galli C: Olive oil: more than just oleic acid. Am J Clin Nutr 72: 853, 2000

15. Owen RW, Giacosa A, Hull WE, Haubner R, Spiegelhalder B and Bartsch $\mathrm{H}$ : The antioxidant/anticancer potential of phenolic compounds isolated from olive oil. Eur J Cancer 36: 1235-1247, 2000.

16. Tuck KL and Hayball PJ: Major phenolic compounds in olive oil: metabolism and health effects. J Nutr Biochem 13: 636-644, 2002.

17. Visioli F, Poli A and Galli C: Antioxidant and other biological activities of phenols from olives and olive oil. Med Res Rev 22: 65-75, 2002.

18. Menendez JA, Vazquez-Martin A, Colomer R, Brunet J, Carrasco-Pancorbo A, Garcia-Villalba R, Fernandez-Gutierrez A and Segura-Carretero A: Olive oil's bitter principle reverses acquired autoresistance to trastuzumab (Herceptin) in HER2overexpressing breast cancer cells. BMC Cancer 7: 80, 2007.

19. Colomer R, Lupu R, Papadimitropoulou A, Vellón L, Vázquez-Martín A, Brunet J, Fernández-Gutiérrez A, SeguraCarretero A and Menéndez JA: Giacomo Castelvetro's salads. Anti-HER2 oncogene nutraceuticals since the 17 th century? Clin Transl Oncol 10: 30-34, 2008.

20. Aggarwal BB and Shishodia S: Molecular targets of dietary agents for prevention and therapy of cancer. Biochem Pharmacol 71: 1397-1421, 2006.
21. Sant M, Allemani C, Sieri S, Krogh V, Menard S, Tagliabue E, Nardini E, Micheli A, Crosignani P, Muti P and Berrino F: Salad vegetables dietary pattern protects against HER-2-positive breast cancer: A prospective Italian study. Int J Cancer 121: 911-914, 2007.

22. Gomez Caravaca AM, Carrasco Pancorbo A, Canabate Diaz B, Segura Carretero A and Fernandez Gutierrez A: Electrophoretic identification and quantitation of compounds in the polyphenolic fraction of extra-virgin olive oil. Electrophoresis 26: 3538-3551, 2005.

23. Carrasco-Pancorbo A, Cerretani L, Bendini A, Segura-Carretero A, Del Carlo M, Gallina-Toschi T, Lercker G, Compagnone D and Fernandez-Gutierrez A: Evaluation of the antioxidant capacity of individual phenolic compounds in virgin olive oil. J Agric Food Chem 53: 8918-8925, 2005.

24. Carrasco-Pancorbo A, Arraez-Roman D, Segura-Carretero A and Fernandez-Gutierrez A: Capillary electrophoresiselectrospray ionization-mass spectrometry method to determine the phenolic fraction of extra-virgin olive oil. Electrophoresis 27: 2182-2196, 2006.

25. Carrasco-Pancorbo A, Gomez-Caravaca AM, Cerretani L, Bendini A, Segura-Carretero A and Fernandez-Gutierrez A: Rapid Quantification of the Phenolic Fraction of Spanish Virgin Olive Oils by Capillary Electrophoresis with UV Detection. J Agric Food Chem 54: 7984-7991, 2006.

26. Carrasco-Pancorbo A, Neusüss C, Pelzing M, Segura-Carretero A and Fernández-Gutiérrez A: CE- and HPLC-TOF-MS for the characterization of phenolic compounds in olive oil. Electrophoresis 28: 806-821, 2007.

27. Bendini A, Cerretani L, Carrasco-Pancorbo A, Gómez-Caravaca AM, Segura-Carretero A, Fernández-Gutiérrez A and Lercker G: Phenolic molecules in virgin olive oils: a survey of their sensory properties, health effects, antioxidant activity and analytical methods. An overview of the last decade. Molecules 12: 1679-1719, 2007.

28. Arenkov P, Kukhtin A and Gemmell A: Protein microarrays: use of immunoassays and enzymatic reactions. Anal Biochem 278: 123-131, 2000 .

29. Haab BB, Dunham MJ and Brown PO: Protein microarrays for highly parallel detection and quantitation of specific proteins and antibodies in complex solutions. Genome Biol 2: RES0004, 2001.

30. Charboneau L, Tory H, Chen T, Winters M, Petricoin EF 3rd, Liotta LA and Paweletz CP: Utility of reverse phase protein arrays: applications to signalling pathways and human body arrays. Brief Funct Genomic Proteomic 1: 305-315, 2002.

31. Menendez JA, Mehmi I, Verma VA, Teng PK and Lupu R: Pharmacological inhibition of fatty acid synthase (FAS): a novel therapeutic approach for breast cancer chemoprevention through its ability to suppress Her-2/neu (erbB-2) oncogene-induced malignant transformation. Mol Carcinog 41: 164-178, 2004.

32. Menendez JA, Ropero S, Mehmi I, Atlas E, Colomer R and Lupu R: Overexpression and hyperactivity of breast cancerassociated fatty acid synthase (oncogenic antigen-519) is insensitive to normal arachidonic fatty acid-induced suppression in lipogenic tissues but it is selectively inhibited by tumoricidal alpha-linolenic and gamma-linolenic fatty acids: a novel mechanism by which dietary fat can alter mammary tumorigenesis. Int J Oncol 24: 1369-1383, 2004

33. Menendez JA, Vellon L, Mehmi I, Oza BP, Ropero S, Colomer R and Lupu R: Inhibition of fatty acid synthase (FAS) suppresses HER2/neu (erbB-2) oncogene overexpression in cancer cells. Proc Natl Acad Sci USA 101: 10715-10720, 2004.

34. Vazquez-Martin A, Colomer R, Brunet J, Lupu R and Menendez JA: Overexpression of fatty acid synthase gene activates HER1/HER2 tyrosine kinase receptors in human breast epithelial cells. Cell Prolif 41: 59-85, 2008.

35. Alli PM, Pinn ML, Jaffee EM, McFadden JM and Kuhajda FP. Fatty acid synthase inhibitors are chemopreventive for mammary cancer in neu-N transgenic mice. Oncogene 24: 3946, 2005 . 